\title{
Aspectos éticos, políticas públicas y nuevos paradigmas en nutrición clínica y metabolismo: desafíos para la investigación
}

\author{
Ethical aspects, public policies and new paradigms in clinical \\ nutrition and metabolism: challenges for research
}

Diana Cárdenas ${ }^{1 *}$, Charles Bermúdez²

https://doi.org/10.35454/rncm.v2n1.053

\begin{abstract}
La investigación en nutrición clínica tiene como objetivo, por un lado, estudiar la relación causal entre la administración de nutrientes artificiales y los desenlaces clínicos y, por otro, la determinación de los niveles requeridos de suplementación para lograr resultados específicos. El objetivo final es modular ciertos procesos inmunológicos y metabólicos para prevenir o tratar la desnutrición asociada a la enfermedad y las comorbilidades relaciona$\mathrm{das}^{(1)}$. Al igual que cualquier ciencia se basa en hechos científicos. Un hecho científico es una hipótesis que ha sido corroborada una vez confrontada a la realidad en un experimento o experiencia específica. El método científico no es especulativo y se fundamenta en hechos reales y verificados ${ }^{(2)}$. Por lo tanto, nos preguntamos: ¿Cómo se estudian los nutrientes artificiales? ¿Cómo se demuestra su beneficio clínico?
\end{abstract}

La nutrición clínica ha utilizado el método científico a través de pruebas de eficacia clínica. El punto de partida son los estudios fundamentales (mechanistic studies o basic research) donde se evalúan, con distintas metodologías (metabolómica, isotopos, etc.) y en diversos niveles (celular, tisular, organismos animales o personas) los mecanismos y el papel que desempeñan los nutrientes artificiales en los procesos metabólicos durante la salud

\footnotetext{
Médico Cirujano, Universidad El Bosque. Profesora Asistente, Facultad de Medicina, Instituto de Investigación en Nutrición, Genética y Metabolismo, Universidad El Bosque, Bogotá., D.C, Colombia. Diploma Universitario de Dietética, Nutrición Clínica y Terapéutica, Hospital Bichat, Universidad Denis Diderot, Paris, France. Magister en Ciencias, Universidad René Descartes, Paris, France. Magister en Metabolismo y Nutrición, Universidad Paris Diderot, Francia. Magister en Relaciones Internacionales, Universidad de Lyon, Francia. Doctorado en Filosofía, Universidad de Franche-Comté, Besançon, Francia. *editor-rmnc@nutriclinicacolombia.org

2 Cirujano, Clínica La Colina y Clinica del Country, Bogotá., D.C, Colombia.
}

y la enfermedad. Posteriormente a través de los ensayos controlados aleatorizados (ECA) es posible evaluar los efectos de las intervenciones con nutrientes artificiales sobre los resultados específicos. Según la medicina basada en la evidencia (Evidence-based medicine, EBM) el estudio debe ser aleatorio, doble ciego y controlado con placebo.

En las ultimas décadas se han logrado grandes avances en la nutrición clínica. Sin embargo, persisten dudas e incertidumbres respecto a ciertos temas cruciales como pueden ser la dosis de proteína requerida por el paciente en estado crítico, la utilización de farmaconutrientes, y los criterios diagnósticos de sarcopenia y de desnutrición, entre otros. Estas dudas son aún más profundas en Latinoamérica ya que las recomendaciones de práctica clínica o ciertos parámetros se basan, en su mayoría, en referencias y guías europeas o norteamericanas que no tienen en cuenta las características propias de la población ni los problemas más relevantes de nuestra práctica clínica.

La doctora Isabel Correia en el editorial invitado “¿Falta de ciencia o mala ciencia?” del presente número cuestiona las razones de estas dudas y pone sobre la mesa aspectos muy importantes como la calidad metodológica de los estudios, el mal uso de los meta-análisis y el interés comercial o efecto marketing (y plantea de manera acertada la necesidad de otro editorial sobre el tema) de algunos temas actuales en nutrición clínica. Por mi parte, cuestiono las dificultades de la investigación en nutrición clínica. ¿Podremos explicar la dudosa o mala calidad de algunos estudios como consecuencia de las características particulares y las dificultades específicas de la nutrición clínica?

Antes de mencionar tres dificultades que considero relevantes y sobresalen en los artículos de este número 
de la revista, es preciso constatar que la mayoría de las recomendaciones de las guías de práctica clínica se basan en niveles de evidencia bajos. Por ejemplo, las Guías de ESPEN de Nutrición en el paciente en cuidado crítico publicadas en 2018 presentan un total de 57 recomendaciones $^{(3)}$. El $41 \%$ son recomendaciones basadas en la experiencia clínica de los participantes en las guías (GPP, Good practice points); 33 \% son de tipo B, es decir, basadas en estudios clasificados como $2++$ (revisiones sistemáticas de alta calidad, estudios casos y controles o estudios de cohorte, ver guía para mayor detalle); $19 \%$ recomendaciones tipo 0 , es decir, estudios no analíticos, series de casos y opinión de expertos. Solo 7 \% de las recomendaciones se basan en una evidencia sólida con ECA de bajo riesgo de sesgo, meta-análisis de calidad y revisiones sistemáticas. Esto significa que las decisiones aún se toman basadas en la intuición, la opinión y la experiencia individual, en otras palabras, de manera especulativa. Las opiniones podrían surgir de aquellas investigaciones fundamentales donde a partir del conocimiento básico sobre los aspectos fisiológicos del nutriente se infiere en un posible efecto benéfico en un estado patológico. Es decir, partiendo del conocimiento de la fisiopatología de la enfermedad y las acciones farmacológicas o metabólicas del nutriente es posible elaborar un racionamiento teórico de tipo "mecanicista" que permite predecir o sugerir un beneficio terapéutico. Sin embargo, estas afirmaciones pueden ser erróneas y muchas veces no ser benéficas; y además, tener efectos secundarios graves. Por ejemplo, los estudios que evalúan las propiedades antioxidantes y las acciones sobre el metabolismo de lípidos de la vitamina $\mathrm{E}$, inducen a pensar que una alimentación enriquecida en esta vitamina podría asociarse a una menor mortalidad cardiovascular. Incluso los estudios observacionales en poblaciones con una alimentación rica en vitamina $\mathrm{E}$ muestran menor mortalidad cardiovascular.

$\mathrm{Si}$ bien estos conocimientos fundamentales son importantes también son fragmentados y el razonamiento teórico que lo sustenta es especulativo. Esto hace que las deducciones en este nivel no puedan garantizar la exactitud del razonamiento y sus conclusiones, en nuestro caso, la eficacia del nutriente o la intervención nutricional. Por lo tanto, los ECA se hacen indispensables, ya que siempre que sean realizados correctamente, son capaces de demostrar un beneficio real. En el caso de la vitamina $\mathrm{E}$, a pesar de ECA bien hechos y con un número importante de pacientes no ha sido posible mostrar beneficio clínico de la administración de la vitamina $\mathrm{E}^{(4)}$.
Veamos el caso de la glutamina (Gln) inmunonutriente de moda en la década de los 90 y 2000 . Estudios en animales han demostrado que mejora la función de barrera intestinal y tiene un efecto en el estrés oxidativo y la inhibición de los procesos inflamatorios como la activación de NF-k $\beta$ y la producción de TNF-a. La cascada de regulación de señal inducida por la Gln la describen los doctores Manzanarez y Hardy en el brillante artículo publicado en el presente número. Ellos describen como la administración de Gln resulta en la supresión de muchos mediadores inflamatorios importantes incluyendo especies reactivas de oxígeno. Por lo tanto, se podría inducir que este nutriente administrado en dosis farmacológicas tendría un efecto benéfico en los pacientes sépticos o con grado severo de inflamación de las unidades de cuidados intensivos (UCI). No obstante, las guías de ESPEN, con grado A de evidencia, recomiendan no administrar Gln en forma de dipéptido en pacientes inestables o difíciles y con falla hepática o renal en la UCI. Los autores del artículo hacen énfasis en la necesidad de conocer los niveles plasmáticos de los inmunonutrientes de los pacientes antes de su suplementación y de adoptar un enfoque más farmacéutico clasificando los inmunonutrientes como medicamentos, es decir, estudiarlos bajo el paradigma de la farmaconutrición.

Este es un ejemplo que demuestra en claro la necesidad de realizar ECA en condiciones apropiadas y teniendo en cuenta algunas particularidades de la nutrición clínica.

\section{ES NECESARIO CONSIDERAR LOS NUTRIENTES COMO VERDADEROS FÁRMACOS}

Los antecedentes de ingesta y los niveles plasmáticos basales del nutriente o inmunonutriente de interés pueden influir en la respuesta que se está estudiando.

En los estudios con fármacos, los investigadores se aseguran de que los participantes en las investigaciones no tengan niveles plasmáticos del fármaco que se estudia. Por el contrario, la mayoría de los estudios en nutrición no consideran los niveles basales de los nutrientes y en ocasiones tampoco se evalúa la ingesta. Se sabe que estos niveles pueden tener influencia en la respuesta esperada. Muchos nutrientes tienen umbrales, es decir, están limitados por la saturación de enzimas, el transportador o el receptor. Además, el metabolismo de muchos nutrientes es distinto en estados hipercatabólicos en comparación con la persona sana, lo que puede conllevar a deficiencias específicas o a niveles basales aumentados. Los 
requerimientos no son los mismos y en el paciente en estado hipercatabólico no se trata únicamente de suplir un déficit o cubrir un requerimiento de nutrientes sino de modular una respuesta metabólica.

En los estudios clínicos, ¿si el nivel de referencia del nutriente es adecuado, se debe esperar que el resultado sea mínimo? Si lo que se desea es un efecto "suprafisiológico", ¿cómo conocer el momento en que se llega a estos niveles si no se conoce el nivel de base? Realizar los estudios bajo el paradigma de la farmaconutrición podría dar respuesta a este punto además de un mejor conocimiento de la respuesta metabólica adaptativa al estrés.

\section{EL PROBLEMA ÉTICO DE LOS GRUPOS CONTROL EN NUTRICIÓN CLÍNICA}

Un grupo control es un conjunto de individuos que no recibirá o no se verá afectado por el tratamiento estudiado y es indispensable para poder evaluar el efecto del tratamiento o la intervención. Algunos individuos pueden ser seleccionados para hacer parte de este grupo, lo cual tiene implicaciones éticas. Por ejemplo: ¿es ético estudiar un grupo de personas con sarcopenia o desnutrición sabiendo que al recibir el placebo no se corrige este estado? ¿Es ético estudiar la administración de dosis altas de proteína en el paciente en estado crítico $(\geq 2,2$ $\mathrm{g} / \mathrm{kg} / \mathrm{d}$ ) como proponen en el estudio clínico EFFORT los autores Ortiz y Heyland en el artículo que se publica en el presente número? En ambos casos, la respuesta es sí. Es decir, sí es ético siempre y cuando se aplique el principio de indeterminación clínica también conocido como incertidumbre terapéutica (clinical equipoise). Los individuos con sarcopenia/desnutrición y los pacientes que recibirán dosis altas de proteínas pueden hacer parte de estos estudios ya que existe duda o incertidumbre sobre la mejor opción o sobre cual debe ser el tratamiento estándar. Este concepto implica que existe una incertidumbre real sobre el beneficio del tratamiento, lo cual se cumple en el caso del estudio EFFORT. Las guías de ESPEN confirman esta duda y con un nivel de evidencia 3 y 4 (opinión de expertos) recomiendan 1,3 $\mathrm{g} / \mathrm{kg} /$ día y afirman que es posible que de igual manera que con los objetivos calóricos, los objetivos óptimos de proteínas en la UCI cambien con el tiempo, y que una ingesta alta de proteínas solo sea beneficiosa si no se asocia a la sobrealimentación (exceso de calorías). Considero que estos últimos aspectos deberían ser abordados por el estudio EFFORT y otros ECA que pretendan elucidar este punto.

\section{LATINOAMÉRICA NECESITA MEJORAR LA INVESTIGACIÓN EN NUTRICIÓN CLÍNICA Y REALIZAR ECA DE BUENA CALIDAD PARA CONSTRUIR SU PROPIA EVIDENCIA Y RECOMENDACIONES}

Aunque podríamos afirmar que la falta de recursos y de educación en este campo son las principales razones para que la región no construya su propio conocimiento, el vacío de políticas públicas es el verdadero punto de partida del problema. En ese sentido, la Declaración de Cartagena sobre el derecho al cuidado nutricional y la lucha contra la desnutrición, que se firmará en la asamblea extraordinaria de la FELANPE en el mes de mayo, podrá contribuir a construir políticas públicas basadas en sus principios fundamentales. El doctor Humberto Arenas expresidente de la FELANPE 20162018 hace una reflexión de la Declaración de Cancún de 2008 sobre el derecho a la alimentación en los hospitales, antecedente importante de la Declaración de Cartagena ${ }^{(5)}$. Además, aplaudimos los esfuerzos de la ACNC por realizar la reunión de consenso colombiano sobre inmunonutrición en el paciente quirúrgico las cuales publicamos en el presente número de la Revista de Nutrición Clínica y Metabolismo.

Aquí no se pone en cuestionamiento la medicina basada en la evidencia, sino que se plantean algunos desafíos y dificultades de la investigación en nutrición clínica y en la necesidad de construir una mejor evidencia científica. Esto podría realizarse teniendo en cuenta la necesidad de considerar los nutrientes como verdaderos fármacos, los aspectos éticos de la definición de los grupos de control y la falta de recomendaciones y de evidencia científica propia en Latinoamérica.

Esperamos que la Revista de Nutrición Clínica y Metabolismo sea un medio que favorezca la comunicación y la construcción del conocimiento en la nutrición clínica de la región; y por ende, mejore la calidad de la atención de los pacientes que requieren nutrición artificial la cual deberá estar basada en la mejor evidencia posible.

\section{Referencias bibliográficas}

1. Cederholm T, Barazzoni R, Austin P, Ballmer P, Biolo G, Bischoff SC. ESPEN guidelines on definitions and terminology of clinical nutrition. Clin Nutr 2017;36:49-64.

2. Lecourt D. La Philosophie des Sciences. Paris, PUF, 2012.

3. Singer P, Reintam Blaser A, Berger MM, Alhazzani W, Calder $\mathrm{Ph}$. Casaer Mp. ESPEN guideline on clinical nutrition in the intensive care unit. Clin Nutr. 2018;1-32. 
4. Gaziano JM. Vitamin E and cardiovascular disease: observational studies. Ann N Y Acad Sci. 2004;1031:280-91.

5. Castillo Pineda JC, Figueredo Grijalva R, Dugloszewski C, Ruy Díaz Reynoso JAS, Spolidoro Noroña JV, Matos A, et al.
Declaración de Cancún: declaración internacional de Cancún sobre el derecho a la nutrición en los Hospitales. Nutr Hosp. 2008;23(5):413-7. 